\title{
Peertechz
}


Short Communication

\section{Dirac spinor's transformation under Lorentz mappings}

\section{J Yaljá Montiel-Pérez ${ }^{1}$, J López-Bonilla ${ }^{2 *}$ and VM Salazar del Moral ${ }^{2}$}

${ }^{1}$ Centro de Investigación en Computación, Instituto Politécnico Nacional, México

${ }^{2}$ ESIME-Zacatenco, Instituto Politécnico Nacional, México
Received: 30 June, 2021

Accepted: 13 July, 2021

Published: 15 July, 2021

*Corresponding authors: J López-Bonilla, ESIMEZacatenco, Instituto Politécnico Nacional, Edif. 4, 1er. Piso, Col. Lindavista CP 07738, CDMX, México,

E-mail: jlopezb@ipn.mx

Keywords: Dirac 4-spinor, Lorentz transformation, Dirac equation, Pauli and dirac matrices.

https://www. peertechzpublications.com

Check for updates

\section{Abstract}

For a given Lorentz matrix, we deduce the Dirac spinor's transformation in terms of four complex quantities.

\section{Introduction}

We have the Dirac equation for spin-1/2 particles [1-5] $\left[\left(x^{\mu}\right)=(t, x, y, z), \hbar=c=1\right]$ :

$$
\left(i \gamma^{\mu} \partial_{\mu}-m_{0}\right) \psi=0, \quad i=\sqrt{-1}, \quad \partial_{\mu}=\frac{\partial}{\partial x^{\mu}},
$$

where $\psi$ is a 4-spinor with the $\gamma^{\mu}$ matrices verifying the anticommutator [6-8]:

$$
\left\{\gamma^{\mu}, \gamma^{\nu}\right\}=2 g^{\mu v} I_{4 x 4}, \quad\left(g^{\mu \nu}\right)=\operatorname{Diag}(1,-1,-1,-1) .
$$

Here we shall use the Dirac-Pauli (or standard) representation [2,9]:

$$
\gamma^{0}=\left(\begin{array}{cc}
I & 0 \\
0 & -I
\end{array}\right), \quad \gamma^{j}=\left(\begin{array}{cc}
0 & \sigma_{j} \\
-\sigma_{j} & 0
\end{array}\right), j=1,2,3,
$$

with the Cayley [10]-Sylvester [11]-Pauli [12] matrices:

$$
\sigma_{1}=\left(\begin{array}{ll}
0 & 1 \\
1 & 0
\end{array}\right), \quad \sigma_{2}=\left(\begin{array}{cc}
0 & -i \\
i & 0
\end{array}\right), \quad \sigma_{3}=\left(\begin{array}{cc}
1 & 0 \\
0 & -1
\end{array}\right),
$$

to analyze the transformation law of $\psi$ under the orthochronic and proper Lorentz group [13-19]:

$$
\tilde{\sim}^{\mu}=L^{\mu}{ }_{v} x^{v},
$$

which implies the existence $[2,7,20,21]$ of a non-singular matrix $S$ such that:

$L^{\mu}{ }_{v} S \gamma^{v}=\gamma^{\mu} S$, 
and we deduce the relativistic invariance of (1) if the Dirac 4-spinor obeys the transformation rule:

$$
\psi=S \psi
$$

Here we exhibit a method to findS for a given Lorentz matrix.

\section{Construction of the matrix S for a given Lorentz mapping.}

The arbitrary complex quantities $\alpha, \beta, \gamma, \delta$ verifying the constraint $\alpha \delta-\beta \gamma=1$, generate a Lorentz matrix $L=\left(L^{\mu}{ }_{v}\right)$ via the relations $[13-15,17,22-26]:$

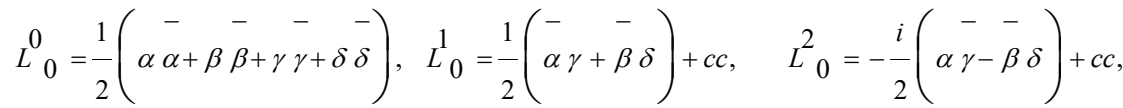



$$
\begin{aligned}
& L_{2}^{0}=-\frac{i}{2}\left(\begin{array}{c}
-\quad- \\
\alpha \beta+\gamma \delta
\end{array}\right)+c c, \quad L_{2}^{1}=-\frac{i}{2}\left(\begin{array}{ll}
- & - \\
\alpha \delta+\beta \gamma
\end{array}\right)+c c, \quad L_{2}^{2}=\frac{1}{2}\left(\begin{array}{l}
- \\
\alpha \delta-\beta \gamma
\end{array}\right)+c c,
\end{aligned}
$$

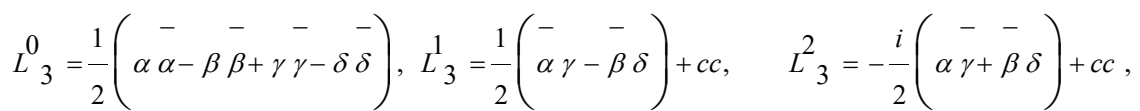



$$
\begin{aligned}
& L_{3}^{3}=\frac{1}{2}\left(\begin{array}{ccc}
- & - & - \\
\alpha \alpha-\beta \beta-\gamma \gamma+\delta \delta
\end{array}\right), \quad \alpha \delta-\beta \gamma=1,
\end{aligned}
$$

where $c c$ means the complex conjugate of all the previous terms.

The inverse problem is to obtain $\alpha, \beta, \gamma, \delta$ if we know $L$, and the answer is [26-29]:

$$
\begin{aligned}
& \alpha=\frac{1}{D} Q_{1}^{1}=\frac{1}{2 D}\left[L_{0}^{0}+L_{3}^{0}+L_{1}+L_{2}^{2}+L_{0}^{3}+L_{3}^{3}-i\left(L_{2}-L_{1}^{2}\right)\right], \\
& \beta=\frac{1}{D} Q_{2}^{1}=\frac{1}{2 D}\left[L^{0}{ }_{1}+L_{0}^{1}-L_{3}+L_{1}^{3}+i\left(L_{2}^{0}+L_{0}^{2}-L_{3}^{2}+L_{2}^{3}\right)\right], \\
& \gamma=\frac{1}{D} Q_{1}^{2}=\frac{1}{2 D}\left[L^{0}{ }_{1}+L_{0}^{1}+L_{3}^{1}-L_{1}^{3}-i\left(L_{2}^{0}+L_{0}^{2}+L_{3}^{2}-L_{2}^{3}\right)\right], \\
& \delta=\frac{1}{D} Q_{2}^{2}=\frac{1}{2 D}\left[L_{0}^{0}-L_{3}^{0}+L_{1}+L_{2}^{2}-L_{0}^{3}+L_{3}^{3}+i\left(L_{2}-L_{1}^{2}\right)\right],
\end{aligned}
$$

where $D^{2}=Q_{1}^{1} Q_{2}^{2}-Q_{2}^{1} Q_{1}^{2}$

From (6) are immediate the expressions $[3,30]$ :

$$
L_{0}^{\mu}=\frac{1}{4} \operatorname{tr}\left(\gamma^{0} S^{-1} \gamma^{\mu} S\right), \quad L_{k}^{\mu}=-\frac{1}{4} \operatorname{tr}\left(\gamma^{k} S^{-1} \gamma^{\mu} S\right), \quad \mu=0, \ldots, 3, \quad k=1,2,3,
$$

that is, if we know $S$ then with (10) we can determine the Lorentz matrix; (10) generates the relations: 


$$
\begin{array}{ll}
L_{0}^{0}=2\left(b_{0}^{2}-b_{1}^{2}-b_{2}^{2}-b_{3}^{2}\right)-1, & L_{1}^{0}=2\left[\left(b_{2} d_{3}-b_{3} d_{2}\right)+i\left(b_{0} d_{1}-b_{1} d_{0}\right)\right], \\
L_{2}^{0}=2\left[\left(b_{3} d_{1}-b_{1} d_{3}\right)+i\left(b_{0} d_{2}-b_{2} d_{0}\right)\right], & L_{3}^{0}=2\left[\left(b_{1} d_{2}-b_{2} d_{1}\right)+i\left(b_{0} d_{3}-b_{3} d_{0}\right)\right], \\
L_{0}=2\left[-\left(b_{2} d_{3}-b_{3} d_{2}\right)+i\left(b_{0} d_{1}-b_{1} d_{0}\right)\right], & L_{1}^{1}=2\left[\left(b_{0}^{2}-b_{1}^{2}\right)+\left(d_{2}^{2}+d_{3}^{2}\right)\right]-1, \\
L_{2}^{1}=2\left[-\left(b_{1} b_{2}+d_{1} d_{2}\right)-i\left(b_{0} b_{3}+d_{0} d_{3}\right)\right], & L_{3}^{1}=2\left[-\left(b_{1} b_{3}+d_{1} d_{3}\right)+i\left(b_{0} b_{2}+d_{0} d_{2}\right)\right], \\
L_{0}^{2}=2\left[-\left(b_{3} d_{1}-b_{1} d_{3}\right)+i\left(b_{0} d_{2}-b_{2} d_{0}\right)\right], & L_{1}^{2}=2\left[-\left(b_{1} b_{2}+d_{1} d_{2}\right)+i\left(b_{0} b_{3}+d_{0} d_{3}\right)\right], \\
L_{2}^{2}=2\left[\left(b_{0}^{2}-b_{2}^{2}\right)+\left(d_{1}^{2}+d_{3}^{2}\right)\right]-1, & L_{3}^{2}=2\left[-\left(b_{2} b_{3}+d_{2} d_{3}\right)-i\left(b_{0} b_{1}+d_{0} d_{1}\right)\right], \\
L_{0}^{3}=2\left[-\left(b_{1} d_{2}-b_{2} d_{1}\right)+i\left(b_{0} d_{3}-b_{3} d_{0}\right)\right], & L_{1}^{3}=2\left[-\left(b_{1} b_{3}+d_{1} d_{3}\right)-i\left(b_{0} b_{2}+d_{0} d_{2}\right)\right], \\
L_{2}^{3}=2\left[-\left(b_{2} b_{3}+d_{2} d_{3}\right)+i\left(b_{0} b_{1}+d_{0} d_{1}\right)\right], & L_{3}^{3}=2\left[\left(b_{0}^{2}-b_{3}^{2}\right)+\left(d_{1}^{2}+d_{2}^{2}\right)\right]-1,
\end{array}
$$

which allow to obtain $L$ if we have the expansion [31]:

$$
S=b_{0} I+i d_{0} \gamma^{5}+b_{1} \sigma^{23}+b_{2} \sigma^{31}+b_{3} \sigma^{12}+\sum_{j=1}^{3} d_{j} \sigma^{o j} .
$$

However, here we have the inverse problem, that is, to obtain $b_{\mu} \& d_{\mu}, \mu=0, \ldots, 3$ verifying (11) for a given Lorentz matrix. Our answer is the following:

$$
\begin{aligned}
& b_{0}=\frac{1}{4}(\alpha+\alpha+\delta+\delta), b_{1}=\frac{1}{4}\left(\begin{array}{l}
- \\
\beta-\beta+\gamma-\gamma
\end{array}\right), b_{2}=\frac{i}{4}(\beta+\beta-\gamma-\gamma), b_{3}=\frac{1}{4}\left(\begin{array}{l}
- \\
\alpha-\alpha+\delta-\delta
\end{array}\right), \\
& d_{0}=\frac{i}{4}(\alpha-\alpha+\delta-\delta), d_{1}=-\frac{i}{4}\left(\begin{array}{l}
- \\
\beta+\beta+\gamma+\gamma
\end{array}\right), d_{2}=\frac{1}{4}\left(\begin{array}{l}
- \\
\beta-\beta+\gamma-\gamma
\end{array}\right), d_{3}=\frac{i}{4}\left(\begin{array}{l}
- \\
\delta+\delta-\alpha-\alpha
\end{array}\right),
\end{aligned}
$$

hence the expressions (8) are deduced if we apply (13) into (11). Besides, with (13) the matrix (12) acquires the structure:

$$
S=\left(\begin{array}{ll}
A & E \\
E & A
\end{array}\right), \quad A=\frac{1}{2}\left(\begin{array}{ll}
- & - \\
\alpha+\delta & \beta-\gamma \\
-\gamma-\beta & \alpha+\delta
\end{array}\right), \quad E=\frac{1}{2}\left(\begin{array}{ll}
- & - \\
\alpha-\delta & \beta+\gamma \\
- & - \\
\gamma+\beta & \delta-\alpha
\end{array}\right) .
$$

Therefore, for a given Lorentz transformation first we employ (9) to determine $\alpha, \beta, \gamma, \delta$, then $S$ is immediate via (14); this approach is an alternative to the process showed in [31] and to the explicit general formula obtained by Macfarlane [30]:

$$
\mathrm{S}=\frac{1}{4 \sqrt{\mathrm{G}}}\left[\mathrm{G} \mathrm{I}+\frac{\mathrm{i}}{2} \varepsilon_{\mu v \alpha \beta} \mathrm{L}^{\mu v} \mathrm{~L}^{\alpha \beta} \gamma^{5}+\mathrm{i} \Gamma\left(\mathrm{L}^{2}\right)-\mathrm{i}(2+\operatorname{tr} \mathrm{L}) \Gamma(\mathrm{L})\right]
$$

$$
\begin{aligned}
& G=2(1+\operatorname{tr} L)+\frac{1}{2}\left[(\operatorname{tr} L)^{2}-\operatorname{tr} L^{2}\right], \quad \operatorname{tr} L=\sum_{\mu=0}^{3} L_{\mu}^{\mu}, \quad \operatorname{tr} L^{2}=\sum_{\nu, \alpha=0}^{3} L_{\alpha}^{\nu} L_{\nu}^{\alpha}, \\
& \Gamma(L)=\sum_{\mu, \nu=0}^{3} L_{\mu \nu} \sigma^{\mu \nu}, \quad \Gamma\left(L^{2}\right)=\sum_{\alpha, \mu, \nu=0}^{3} L_{\mu \alpha} L^{\alpha}{ }_{\nu} \sigma^{\mu \nu},
\end{aligned}
$$

however, the possible physical applications are not evident in it. In our procedure, for example, the relations (9) are of great interest for the physicists working on supersymmetry [29], and the expressions (14) are very useful to study the relativistic motion of a classical point particle [28]. 


\section{Conclusion}

The Dirac equation is relativistic if the corresponding 4-spinor verifies the transformation (7) under Lorentz mappings, with the matrix $S$ satisfying the condition (6). Here we showed a procedure to construct $S$ for a given Lorentz matrix.

\section{References}

1. Dirac PAM (1928) The quantum theory of the electron. Proc. Roy. Soc. London A117: 610-624 and A118: 351-361. Link: https://bit.ly/2ThErAQ

2. Leite-Lopes J (1977) Introduction to quantum electrodynamics. (Trillas, Mexico).

3. Ohlsson T (2011) Relativistic quantum physics. (Cambridge University Press, England).

4. Bagrov VG, Gitman D (2014) The Dirac equation and its solutions. (Walter de Gruyter GmbH, Berlin).

5. Maiani L, Benhar O (2016) Relativistic quantum mechanics. (CRC Press, Boca Raton, FI, USA).

6. Good RH (1955) Properties of the Dirac matrices. Rev Mod Phys 27: 187-211. Link: https://bit.ly/3khDfsc

7. López-Bonilla J, Rosales L, Zúñiga-Segundo A (2009) Dirac matrices via quaternions. J Sci Res (India) 53: 253-255. Link: https://bit.ly/3idWv77

8. López-Bonilla J, Ovando G (2021) Arbitrary 4x4 matrix in terms of Dirac matrices. Studies in Nonlinear Sci 6: 17-18. Link: https://bit.ly/3B2N1Et

9. Cohen-Tannoudji C, Dupont-Roc J, Grynberg G (1989) Photons and atoms: Introduction to quantum Electrodynamics. (John Wiley and Sons, New York) Chap. 5.

10. Cayley A (1858) A memoir on the theory of matrices. London Phil Trans. 148: 17-37. Link: https://bit.ly/3rbam26

11. Sylvester J (1884) On quaternions, nonions and sedenions. John Hopkins Circ 3: 7-9. Link: https://bit.ly/36BWuVI

12. Pauli W (1927) Zur quantenmechanik des magnetischen electrons. Zeits. für Physik 43: 601-623. Link: https://bit.ly/3hFPJbh

13. Synge JL (1965) Relativity: the special theory. (North-Holland, Amsterdam).

14. López-Bonilla J, Morales J, Ovando G (2002) On the homogeneous Lorentz transformation, Bull. Allahabad Math. Soc. 17: 53-58. Link: https://bit.ly/3B2NeHL

15. Ahsan Z, López-Bonilla J, Man-Tuladhar B (2014) Lorentz transformations via Pauli matrices. J of Advances in Natural Sciences 2: 49-51. Link: https://bit.ly/3ehmc5G 16. Carvajal B, Guerrero I, López-Bonilla J (2015) Quaternions, $2 \times 2$ complex matrices and Lorentz transformations. Bibechana 12: 30-34. Link: https://bit.ly/3B4YseJ 17. López-Bonilla J, Morales-García M (2020) Factorization of the Lorentz matrix. Comput Appl Math Sci 5: 32-33. Link: https://bit.ly/3B4aZiE

18. López-Bonilla J, Morales-Cruz D (2020) Rodrigues-Cartan's expression for Lorentz transformations. Studies in Nonlinear Sci 5: 41-42. Link: https://bit.ly/3rclgVr

19. López-Bonilla J, Morales-Cruz D, Vidal-Beltrán S (2021) On the Lorentz matrix. Studies in Nonlinear Sci 6: 1-3. Link: https://bit.ly/3B2N1Et

20. Pauli W (1936) Contributions mathématiques a la théorie de Dirac. Ann Inst H Poincaré 6: 109-136. Link: https://bit.ly/2UlwDbA

21. Rose ME (1961) Relativistic electron theory. (John Wiley and Sons, New York).

22. Rumer J (1936) Spinorial analysis. (Moscow)

23. Aharoni J (1959) The special theory of relativity. (Clarendon Press, Oxford).

24. Penrose R, Rindler W (1984) Spinors and space-time. I. (Cambridge University Press).

25. Acevedo M, López-Bonilla J, Sánchez M (2005) Quaternions, Maxwell equations and Lorentz transformations. Apeiron 12: 371-384. Link: https://bit.ly/3ehmPMA

26. Cruz-Santiago R, López-Bonilla J, Mondragón-Medina N (2021) Unimodular matrix for a given Lorentz transformation. Studies in Nonlinear Sci 6: 4-6. Link: https://bit.ly/3B2N1Et

27. Gürsey F (1955) Contribution to the quaternion formalism in special relativity. Rev Fac Sci Istanbul A20:149-171. Link: https://bit.ly/3wQSVW9

28. Gürsey F (1957) Relativistic kinematics of a classical point particle in spinor form. Nuovo Cim. 5: 784-809. Link: https://bit.ly/2TdnW8T

29. Müller-Kirsten H, Wiedemann A (2010) Introduction to supersymmetry. (World Scientific, Singapore).

30. Macfarlane AJ (1966) Dirac matrices and the Dirac matrix description of Lorentz transformations. Commun Math Phys 2: 133-146. Link: https://bit.ly/3rcsngP

31. Caicedo-Ortiz HE, López-Bonilla J, Vidal-Beltrán S (2021) Lorentz mapping and Dirac spinor. Comput. Appl Math Sci 6: 9-13. Link: https://bit.ly/2UPIGDP

Copyright: () 2021 Montiel-Pérez JY, et al. This is an open-access article distributed under the terms of the Creative Commons Attribution License, which permits unrestricted use, distribution, and reproduction in any medium, provided the original author and source are credited.

Citation: Montiel-Pérez JY, López-Bonilla J, Salazar del Moral VM (2021) Dirac spinor's transformation under Lorentz mappings. Ann Math Phys 4(1): 028-031. DOI: https://dx.doi.org/10.17352/amp.000022 